\title{
On the Effects of Viewing Cues in Comprehending Distortions
}

\author{
A. Zanella, M.S.T. Carpendale, M. Rounding \\ Department of Computer Science, University of Calgary, \\ 2500 University Dr NW, Calgary, AB., \\ \{azanella,sheelagh,rounding\}@cpsc.ucalgary.ca
}

\begin{abstract}
As a community, human-computer information and interface designers have tended to avoid use of fisheyes, and multi-scale presentations with their attendant distortion because of concern about how this distortion may lead to confusion and misinterpretation. On the other hand, for centuries, hand-created information presentations have made regular use of distortion to provide emphasis and actually enhance readability. Is the lack of use in computer presentations because thus far in our computational uses of distortion we have failed to provide adequate support that allows people to comprehend the manner in which the information is being presented? We describe a study about relative difficulty in reading distortions that investigates the effect of the use viewing cues such as the cartographic grid and shading on people's ability to interpret distortions. We look at two interpretation issues: whether people can locate the region of magnification and whether people can read the relative degree of magnification of these regions. We present the findings of this study and a discussion of its results.
\end{abstract}

\section{Keywords}

User study, distortion viewing, viewing cues, information visualisation, detail-in-context

\section{INTRODUCTION}

Much of the information that is presented to us on paper is distorted. Take for example maps. To begin with all maps are distorted as data that exists on a sphere is projected unto a flat paper. In maps, great care is taken to protect the viewer from misinterpretations that might arise from these distortions. This is most commonly done by the inclusion of latitude and longitude lines. These lines make the projection and therefore the distortion that has been used explicit. In contrast, while many types of distortion have been suggested as possible solutions to the screen real-estate problem, little to no attention has been paid to providing some type of aid to help viewers interpret them.

The screen real-estate problem can be stated, as no matter what size your display is it seems that it is never big enough. This comment has been made in reference to wall size displays, common desktop and laptop displays and of course the increasingly common hand held displays. Lack of space and issues of spatial organisation arise whether one is viewing a large single image or map, coping with multiple files when editing or coding, or trying to organise the windows and icons that are necessary for one's current task. This discrepancy between a computer's display space and its information space is associated with problems in navigation, and interpretation (Leung and Apperley 1994).

One approach that has been suggested for coping with these spatial organisation problems is the creation of multi-scale views. These multi-scale views integrate more than one scale in a given presentation, which leads to the use of some type of distortion. This is not surprising since traditionally 'distortions' have often been used to address problems of fitting information into a given space and to provide the desired information emphasis. For example, many illustrations and diagrams carefully present selected regions of information subtly enlarged to better elucidate the chosen message. Perhaps the general discomfort with the use of distortion is due to the lack of awareness of its widespread use in other presentation mediums. More likely it is due to the fact that on a computer we are faced with interactive distortions and therefore have to support the interpretation of changing distortions.

To better understand these issues and to look at alternatives to solve them, we ran a user study. In this study we are interested in the meta-question of 'to what extent do users need to be protected from mis-interpretation of distorted information?' We examine the effect of adding viewing support by means of visual cues. The viewing cues tested were a grid, shading and the grid and shading combined. 
The study used a matched pair methodology in which a given viewing cue was always matched with a no cue condition (Figure 1). Our basic findings are that including visual cues definitely helps people understand how the information has been presented. People were significantly more accurate and faster when any of the three cues were used. Also, there are differences between the viewing cues that are of interest.

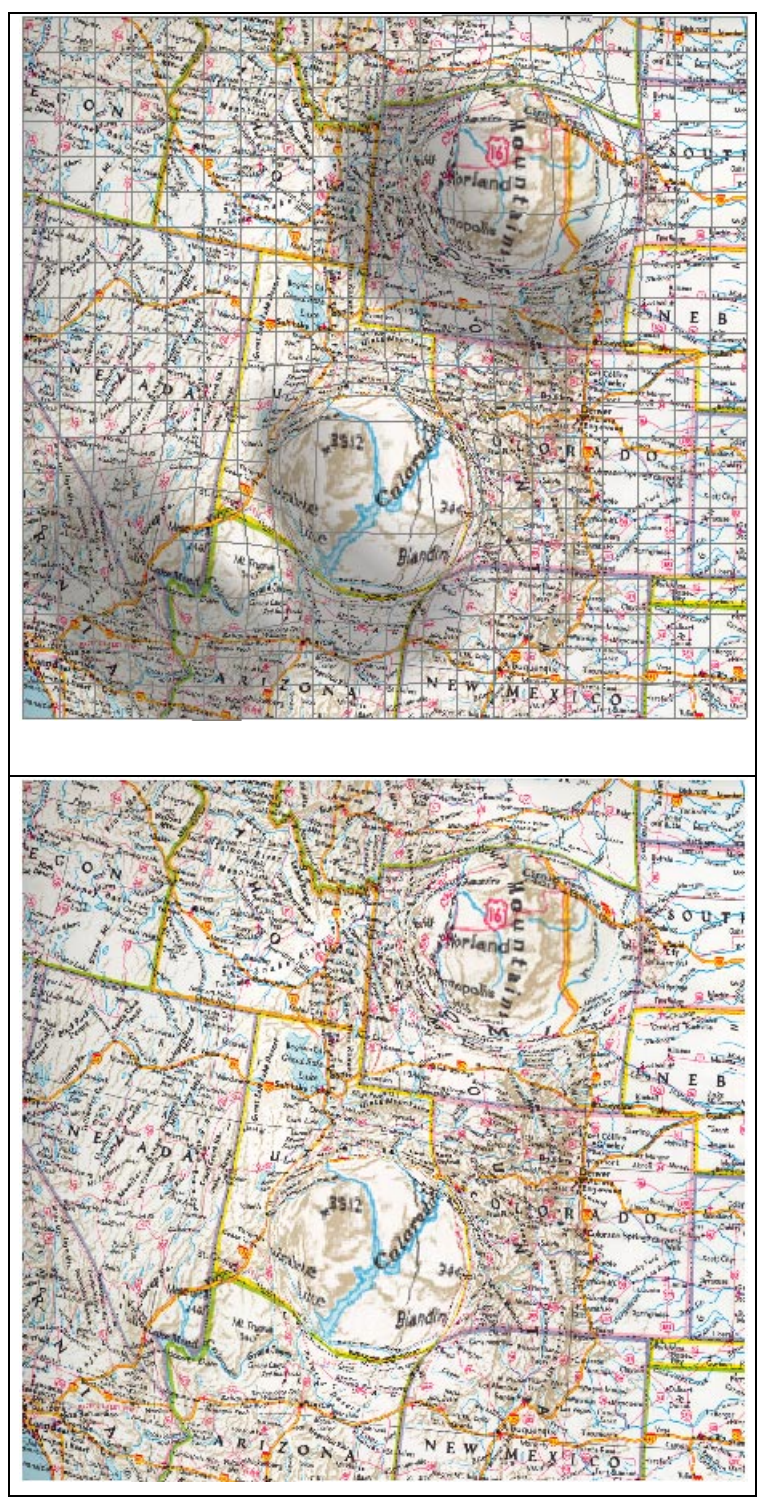

Figure 1: A matched pair of images. The top image has the combined cue of grid and shading. The bottom image has no viewing cue

\section{RELATED WORK}

Research into more effective use of current displays has been categorised as either distortion based or non-distortion based (Leung and Apperley 1994). Non-distortion based screen real estate research has led to most of the more frequently used computational presentation paradigms such as windows with pan, scroll and zoom (for survey see Plaisant et al. 1995). Many researchers have noted limitations of access through pan, scroll and zoom, such as getting lost in information spaces (Meister 1989), problems with maintaining context when examining information details and interpretation issues in comparisons across disparate information spaces (Carpendale et al.1997, Furnas 1986, Spence and Apperley 1982).

Distortion or fisheye based presentation approaches were pioneered by Bifocal Display (Spence and Apperley 1982) and Furnas' paper on Generalized Fisheyes (Furnas 1986). Subsequently several presentation methods have been developed [Bartram et al. 1995, Carpendale et al. 1995, Hamel et al. 1996, Sarkar and Brown 1994, Sarkar et al. 1993) that create displays that vary considerably visually and algorithmically (for surveys see Leung and Apperley 1994, Noik 1994). Research towards the development of detail-in-context methods has concentrated on visual capabilities, such as the number and type of foci. These techniques are said to support human potential for visual gestalt, to reduce cognitive effort needed for the re-integration of information across separate views and to address navigational problems by accessing spatial reasoning (Carpendale et al. 1997, Furnas 1986, Spence and Apperley 1982). Also, studies have indicated that setting detail in its context is common practice in human memory patterns (Furnas 1996). However, comparatively few studies have investigated the use of distortion. In one such study, Schaffer et al. (1993) report a significant advantage for fisheyes in a path finding task. Other studies (Hollands et al. 1989, Kuederle et al. 2001, Storey et al. 1997) have less definite results but do find user preference in favour of the fisheye presentations.

However, there has not been widespread acceptance. While comprehension problems led to the creation of distortion presentations, new comprehension issues have arisen. These include problems recognising that the altered presentations hold the same information (Misue et al. 1995), problems interpreting the information in its distorted form (Carpendale et al. 1997), problems with user disorientation (Hollands et al. 1989, Sarkar and Brown 1994). There have also been comments about how distortions may interfere with the user's mental map (Carpendale et al. 1995, Misue et al. 1995, Storey and Müller 1995).One suggested solution is to limit the type of distortion in an effort to preserve the users mental map by maintaining orthogonality, proximity and topology (Misue et al. 1995). In this context preserving orthogonality means maintaining the left-right and up-down ordering. Maintaining proximity involves preserving distance relationships between objects. 


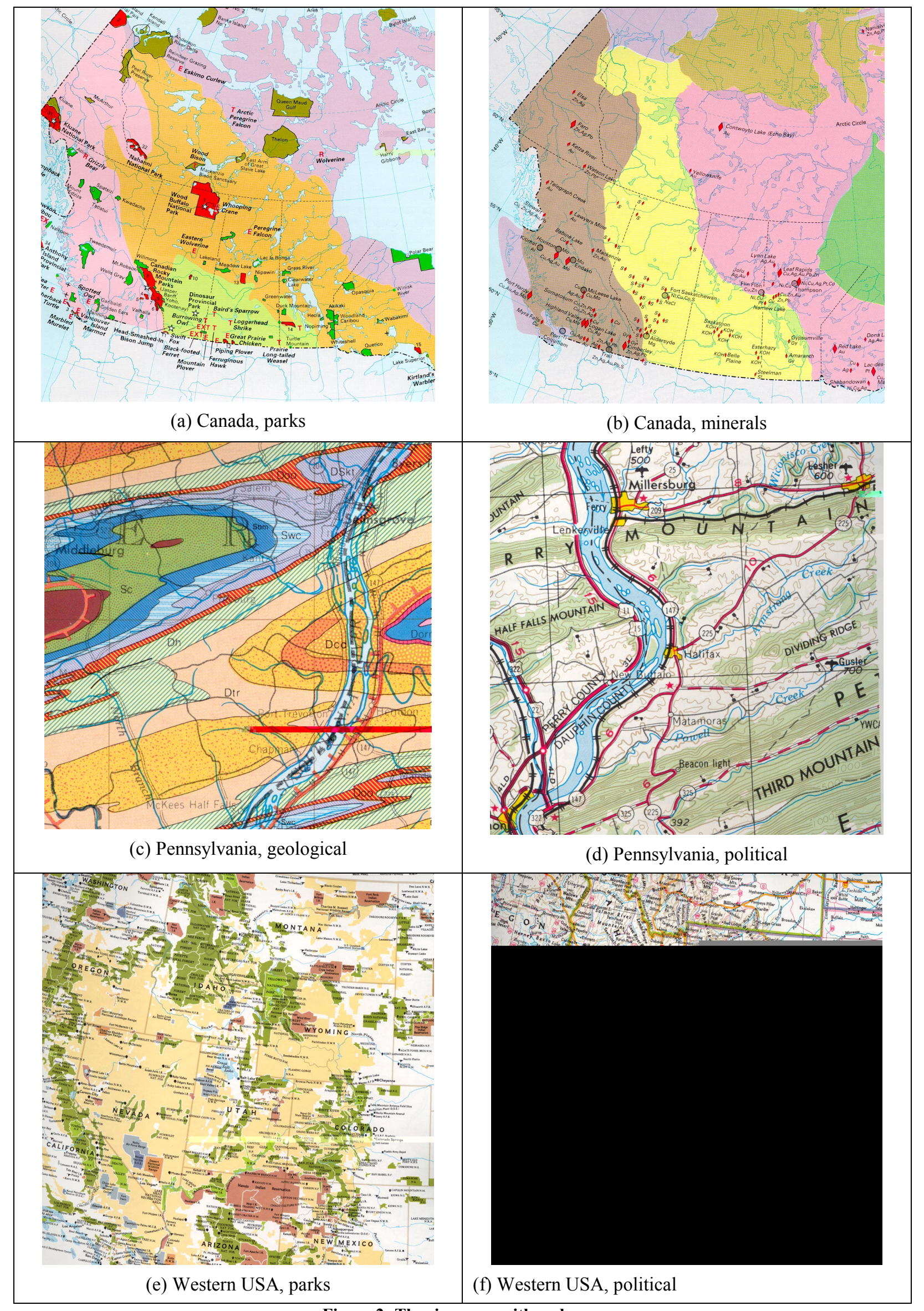

Figure 2: The six maps, with no lenses 
Preserving topology is taken to mean preserving inside-outside relationships. Subsequently it has been noted that a choice may have to be made between preserving proximity and preserving orthogonality (Storey and Muller 1995. Van der Heyden et al. (1999) continue this line of reasoning. Another suggestion is to use visual cues to support users' comprehension of distorted presentations (Carpendale et al. 1997). It is this claim, which has not been previously studied, that we investigate.

\section{EMPIRICAL STUDY}

It would seem that to accrue the benefits in the many claims that have been made about the advantages of distortion viewing, we must learn how to adequately support the user in order to protect them from possible misinterpretations. As a first step, if the user is to be able to adjust their readings to account for the use of distortion the user must be able to:

- tell if there is a distortion present,

- find where this distortion is located, and

- at least be able to have some idea as to the degree of distortion that has been used.

Since the nature of the information representation may also affect people's ability to interpret the lenses we chose to study to one general representation type, maps, and limited the number of map variations to six. These six represent a range of common map types (see Figure 2).

Since we are interested in whether one can comprehend a distortion presentation in general, we start with a worst-case scenario. To this end, we will show users static distortions since the motion or animation of a distortion makes it relatively easy to recognise, and we will show users information that they are not familiar with. Also, since most suggested distortion variations have been presented without any additional visual support, we will show all users all distortions at least once with no viewing cues.

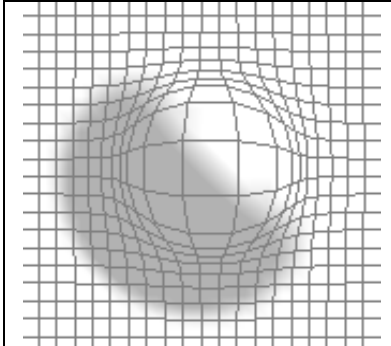

(a) 1 lens

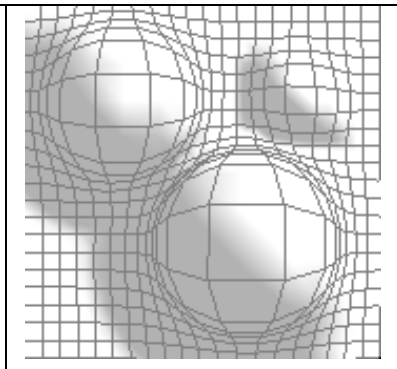

(b) 3 lenses
Figure 3: Distortion detail-in-context lenses shown with both grid and shading

For this study we use constrained detail-in-context lenses, which are fairly typical fisheye distortions that provide space for a region of increased magnification by compressing the immediate surrounds (Figure 3 (a)). We use three sizes of lenses with magnification factors of two, four and six (Figure 3(b)).

Given the six maps (Figure 2), and the three lenses (Figure 3(b)), we examine the addition of visual cues. The term visual cue is used to indicate any aspect of the display that has been added for perceptual reasons, such as attracting attention, creating emphasis, or adding explanation, rather than to directly represent some aspect of the information. In our study we compare images with no visual cues (Figure 4) with the same images with one of three visual cue conditions: the grid (Figure 5(a)), the shading (Figure 5(b)) or the grid and shading combined (Figure 5(c)). This is a matched pair design in that each participant sees the same image twice, once without cues and once with one of the cue conditions.

For each map we created three distorted presentations, with one, two and three lenses, respectively. For each of these presentations we made an image with no cues and one with each of the visual cue conditions: grid, shading, and grid plus shading. We then paired the no cue condition with each of the cue conditions giving three sets of thirtysix images. Each of these three sets were made to contain six grid cues, six shading cues, six grid plus shading cues and their eighteen matching no cue conditions. Since we had thirty participants each set was used ten times each.

We recruited thirty participants, fifteen males and fifteen females, whom were mostly computer science students in undergraduate and graduate levels. Other participants included graduate students in other areas.

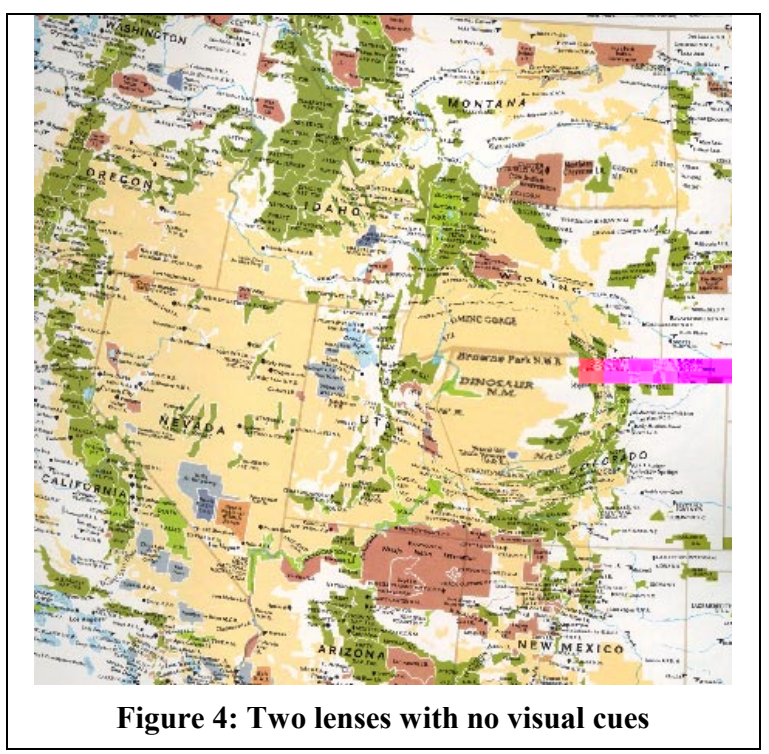




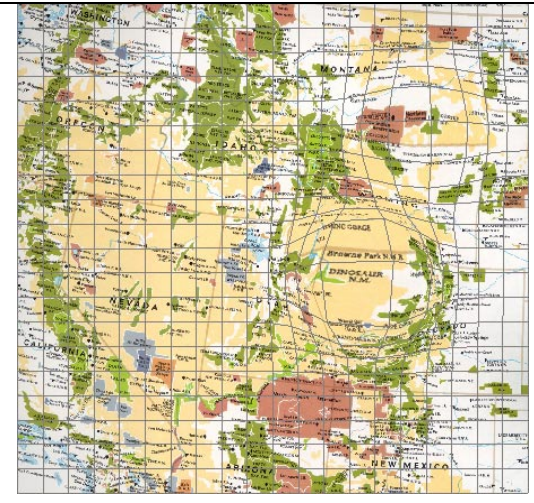

(a) The grid

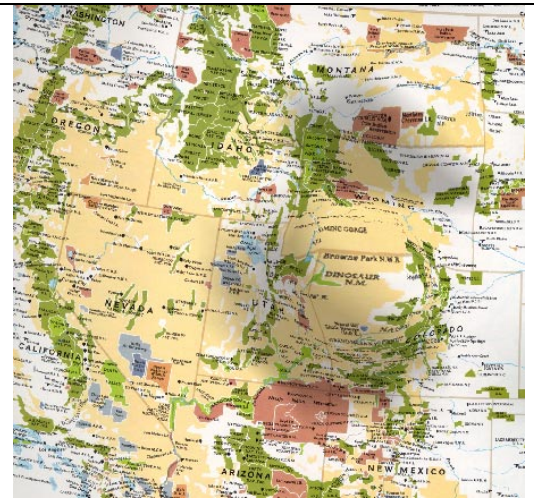

(b) The shading

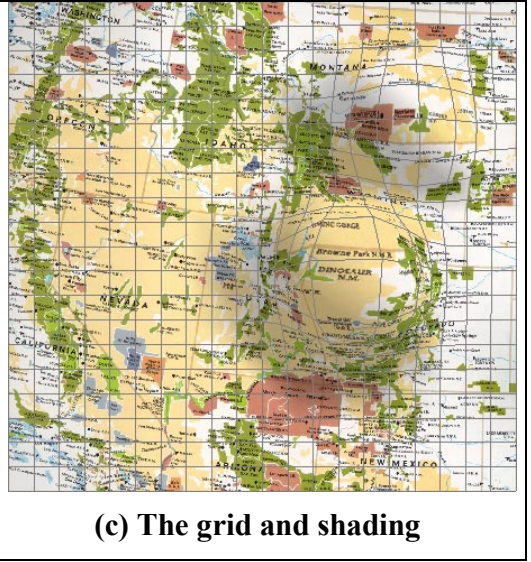

(c) The grid and shading

Figure 5: Three visual cue conditions applied to the map, Canada, minerals: with the same two lenses in each image

\section{Experimental Task}

Thirty-six images were presented to each participant, each image containing from one to three lenses. Each lens configuration on a given map was shown twice: once with no cues and once with one of the three visual cues (see Figure 5 for examples). For each participant the entire sequence of images was randomised. This randomization of the ordering was done within the chosen set of thirty-six images.

Our question is whether visual cues can help protect a user from the possibly being misinformed when they do not realise the presence of a distortion. We asked the participants to tell us if they noticed any distortions by clicking approximately in the focal centre, or the most magnified region of the distortions. Also we asked, if there was more than one region of magnification, that they click on the largest and most magnified first, the second largest next and so on in decreasing size. We referred to this as ranking the lenses. Participants indicated the location of the lens by clicking in the centre of the area of maximum magnification and the rank or their impression of relative degree of magnification by the order in which they clicked on the lenses.

\section{Procedure and Data Collection}

Participants first filled out a background questionnaire that assessed their experience with computers, digital images, maps, distortion lenses and GIS systems. Based on the answers to this initial questionnaire the participants were divided into two groups. In one group the participants had little or no experience with distortion lenses and in the other group the participants that had seen such lenses before. While all participants received a thorough explanation of the distortion lenses and experimental task with the aid of a visual tutorial, we emphasised the difference in experience by giving the more experienced group a training session. This training session gave them approximately twenty minutes to familiarise themselves with lenses in an interactive setting. They were shown how to create lenses on maps and how to interact with them by changing their location, magnification and applying different visual cues. The goal was to provide the participants who had received the benefit of this short training session with a clear understanding of how the lenses and the visual cues behaved in an interactive sense. We were interested in whether the participants could more easily identify the magnification lenses if they had more experience with the concept.

Then they were asked perform the experimental task, finding and ranking the lenses in the images in descending order of magnification. The software tracked the participants' choices as to locations, recorded the distance to the centre of the nearest lens and kept track of time used.

At the end, we asked participants to perform the same experimental task with a small set of images using a talk aloud procedure. Here they performed the same task but explained to the experimenter their process and strategies. These sessions were video taped. Then participants filled out a post-session questionnaire where they indicated their impressions and preferences in terms of locating and ranking the lenses. Additional space was provided for comments.

The independent variables are: the visual cue condition (grid, shading and grid plus shading, see Figure 4 and 5); and maps or information representation (Canada-minerals, Canada-parks, Pennsylvania-geological, Pennsylvania-political, Western US-parks, Western US-political, see Figure 2).

The dependent variables are the location information, the rank order, and the time used. For location information we kept track of the $(\mathrm{x}, \mathrm{y})$ point that was selected, the location of the closest lens, and the distance to the closest lens. Further, we also recorded whether the point selected was in the focal 


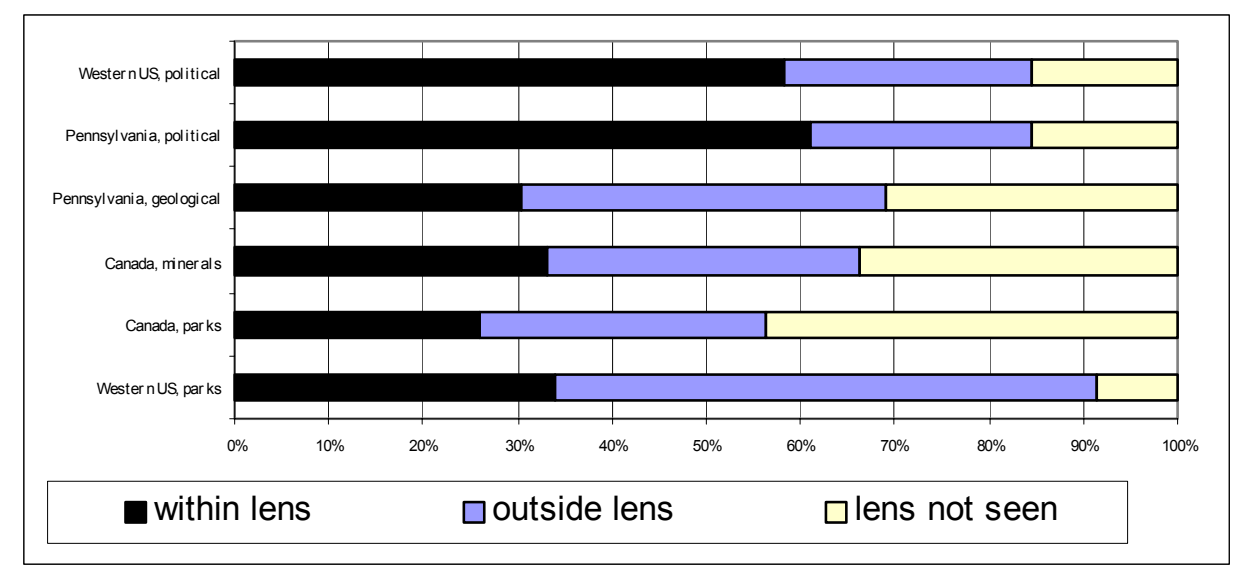

Figure 6: Differences in representation types

region, in the periphery of the focal region, within the lens or elsewhere in the image but just closer to this lens than any other lens. Relative rank information for the lenses within an image was also tracked. There is no rank information for images with only a single lens. Also, the time taken to find each lens was recorded. Qualitative data was also collected; we asked users about their preferences for the visual cues when locating and ranking the lenses.

\section{RESULTS AND DISCUSSION}

\section{The Maps: differing representations}

First we look at whether in the no cue condition, participants had differing degrees of difficulty in locating lenses depending on which map was used. As can be seen in Figure 6 there are quite striking differences. Both the political maps, Pennsylvaniapolitical and Western US-political seem to pose the similar level of difficulty. These political maps have a considerable amount of text and roads and boundaries. Similarly both the categorical maps, Canada-minerals and Pennsylvania-geological, produce comparable results. In contrast, the results for the two parks maps are significantly different from each other and from the other maps. With the Canada-parks map, by far the most lenses are entirely missed, while with the Western US parks map the least number of lenses are actually missed. One surprise is that the two Canadian maps were the ones where people had more difficulty locating lenses, although all subjects were people living in Canada. Since the differing representations where found to affect the results, the rest of the data exploration was conducted with the matched pair data. This means that we are comparing the same maps with the same distortion with the no cue and then a visual cue condition.

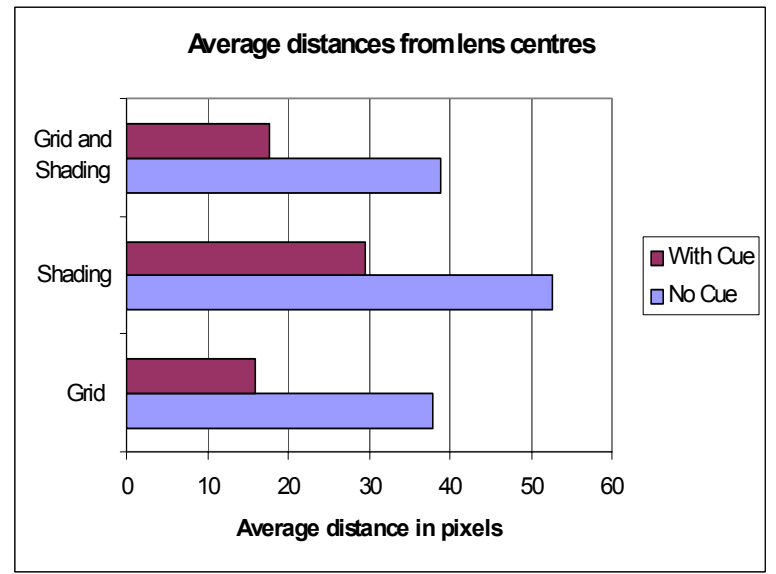

Figure 7: The chart shows the paired conditions (grid \& shading - no cue, shading - no cue and grid - no cue). Notice how even though all of the conditions are helpful there is a considerable difference between the average distances for the cue conditions.

\section{Locating Lenses and Visual Cues}

For the task of locating the lenses, all the visual cues improved the situation significantly (single tail, paired t-test, $\mathrm{p}<0.001$ ). Figure 8 , shows the scatter plots of the actual mouse clicks normalized to set the centre of all regions of magnification at $(100,100)$. Horizontally these images represent the match pairs. The images used for the no cue scatter plots on the left are the exactly the same images as those on the right expect for the addition of the visual cue. Each point represents a location where a participant clicked when locating a lens. For the purposes of comparison, the centre point for each lens has been placed at $(100,100)$. The pattern of dots spatially shows the relative accuracy. As indicated by the spread of locations, it can be seen from these scatter plots that all visual cues helped participants to locate the lenses on maps when compared to the no-cues situation, on the left side. The cues that gave best support were the grid and the shading and grid 
combined, respectively (Figure $8 \mathrm{~b}$ and $8 \mathrm{f}$ ). The missed lenses are not shown in Figure 8. Figure 7 shows the average distances of the mouse clicks from lens centres. These show that while all cue conditions help the participants to more accurately comprehend

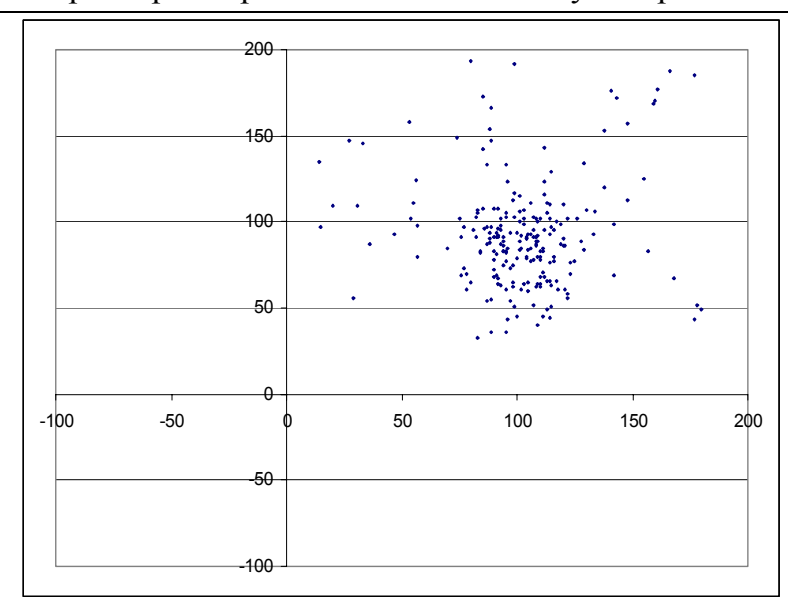

(a) no cues corresponding to grid only images

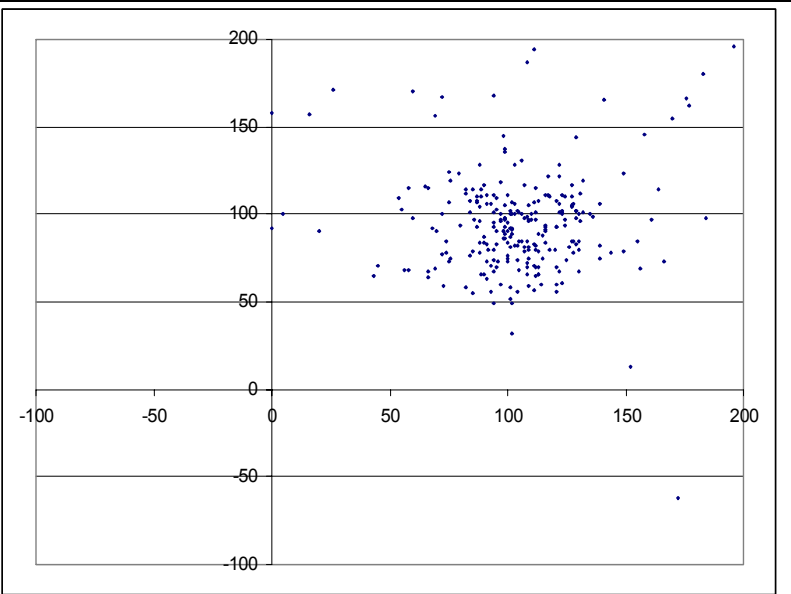

(c) no cues corresponding to shade only images

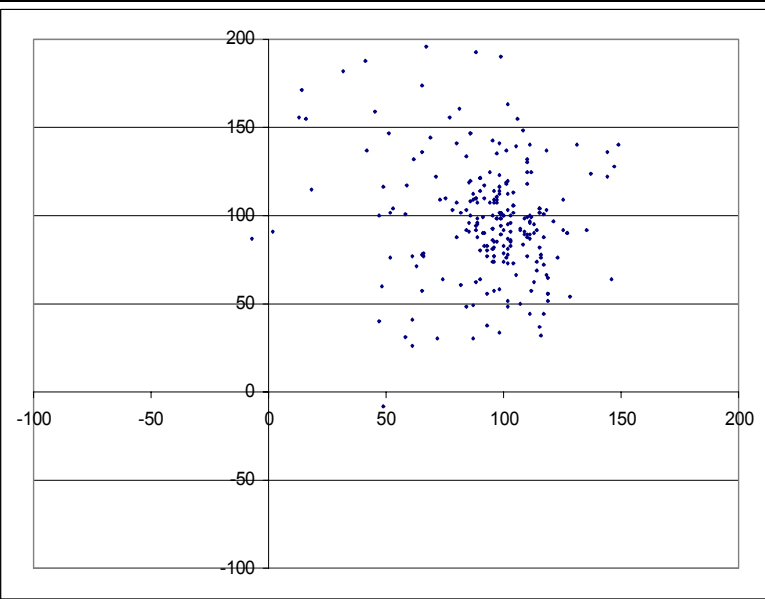

(e) no cues corresponding to shade and grid images the multi-scale presentation there are differences between them. People are really quite accurate with the grid (approximately fifteen pixels from the lens centre) and slightly less accurate with the grid and shading and the least accurate with the shading alone.

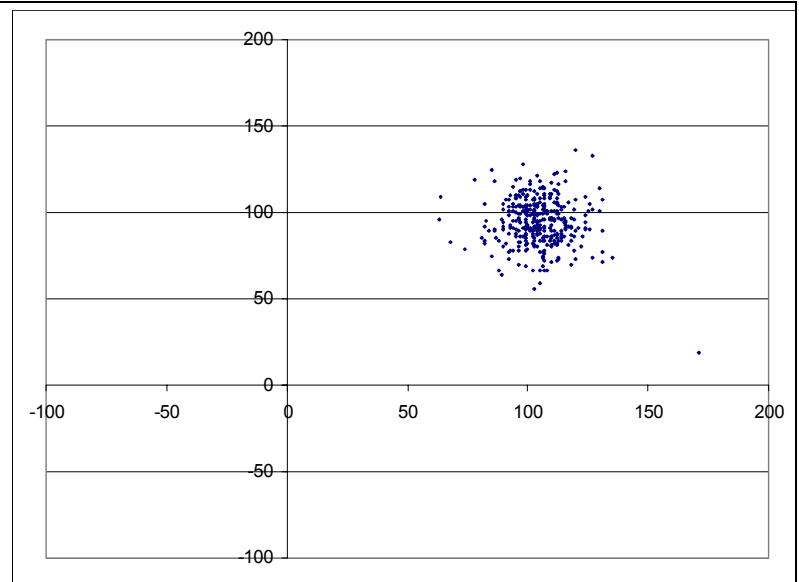

(b) grid

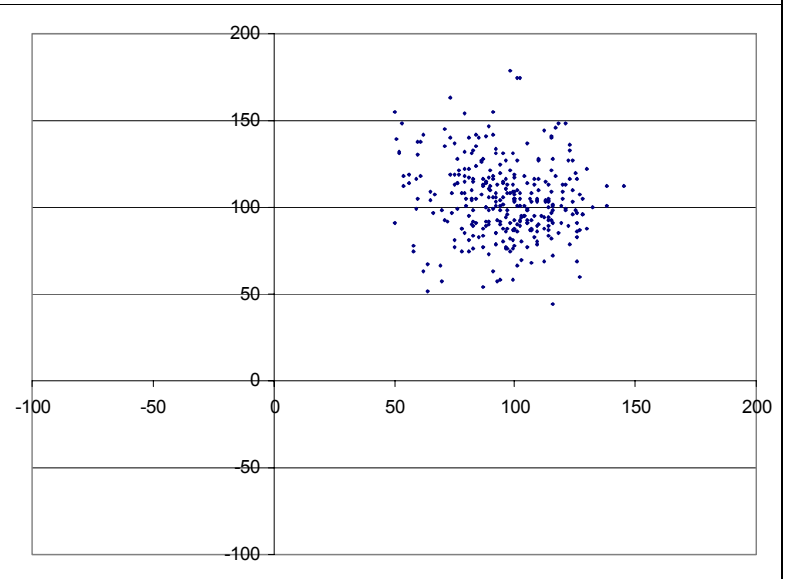

(d) shading

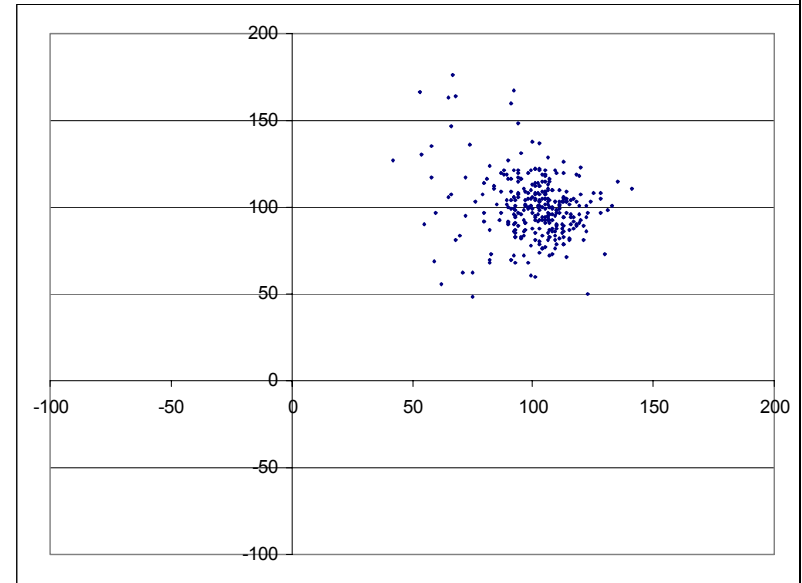

(f) shade and grid

Figure 8: Location accuracy 
Figure 7 shows the average distances of the mouse clicks from lens centres. These show that while all cue conditions help the participants to more accurately comprehend the multi-scale presentation there are differences between them. People are really quite accurate with the grid (approximately fifteen pixels from the lens centre) and slightly less accurate with the grid and shading and the least accurate with the shading alone.

\section{Ranking Lenses and Visual Cues}

Ranking errors were counted by lens. For instance, if the lenses were ordered so that the smallest was indicated to be the largest and the largest was indicated to be the smallest (large, middle, small ordered as small, middle, large), this would be counted as two ranking errors since the middle one is where it should be. No ranking errors were possible if there was only one lens present. Other errors that were also counted are missed lenses and extra lenses. Extra lenses are those that were indicated by a participant when all existing lenses had already been selected. Figure 9 shows these errors as percentages of the possible errors

With the no cue condition approximately a quarter of the lenses are not noticed and of those that are noticed approximately a quarter are mis-ranked. With the grid or the grid plus shading very few lenses are missed and considerably fewer are mis-ranked. The surprising result is that shading provides very little support for the judging of relative magnification, in fact it may actually be misleading. The overall error count for ranking errors with shading is close to fifty percent.

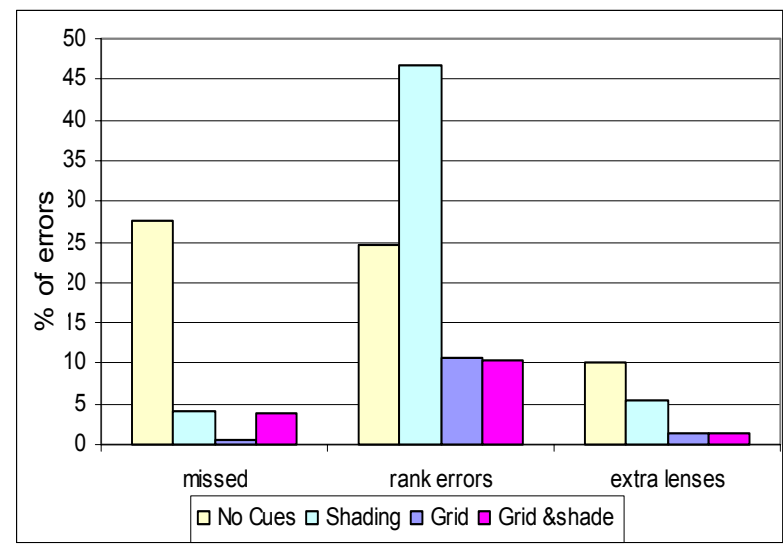

Figure 9: Contrasting the different error types under the different visual cue conditions

\section{Participants Styles and Preferences}

During the debriefing session, we asked the users to go through a set of six to ten more images while relating out loud their thoughts and actions while finding and ranking the lenses. Sometimes we prompted the users for more information with questions such as: "Why do you think it is easy to find the lens in this image?" or, "What type of cue do you first look for in an image?" In this debriefing we wanted to capture the participants' strategies as a more qualitative type of data as compared to what they did as measured by the software. The participants' preferences were recorded in the postsession questionnaire. Figure 10 illustrates the results of this questionnaire. The grid by itself was most preferred by our group of participants for the purposes of both locating and ranking lenses.

For the purpose of locating lenses, the grid cue was preferred for sixteen participants, followed by grid plus shading which was named as the preferred cue for thirteen participants. Only a single participant pointed to shading only as their preferred cue for finding lenses.

For ranking, the grid was clearly the preferred cue with twenty participants choosing it over only eight participants who chose grid and shading and the single participant who chose shading. Interestingly, there was also one participant who chose no cues in this category. The participant explained this by saying that they were looking for distortions of word sizes to help them ascertain the different magnifications.

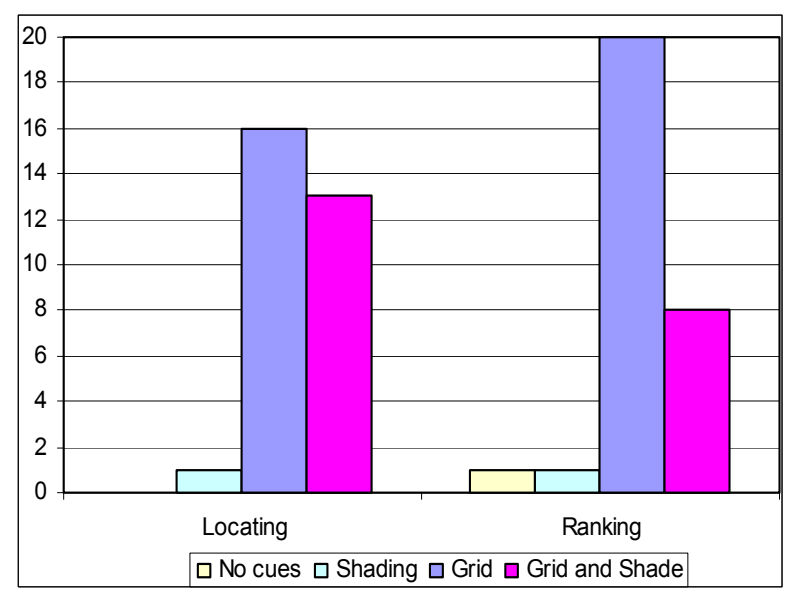

Figure 10: Preferences in locating and ranking lenses

\section{Learning Effects}

There was no significant learning effect resulting from our training sessions in any of the conditions except for shading. The participants who had received a training session made use of the shading cue much more accurately (t-test, trained and not trained, shading cues only, $\mathrm{p}=0.001)$. This is a surprising result. The ability to perceive shape from shading is considered to be a pre-attentive ability (Ramachandran 1988). The fact that the people were getting better at reading the shading cue suggests that some further investigation would be interesting. For our shading visual cue we used a common graphic 
shading algorithm, Gouraud shading (Foley et al.1996). It would appear that this shading algorithm may not have been as effective as was hoped in providing people with the perception of shape from shading. Figure 11 shows one possible explanation of this. The top image shows a faceted cylinder. The chart below shows on the left the luminance profile provide by each of the shading algorithms: uniform shading, Gouraud shading and Phong shading. All of these shading algorithms adjust the resulting image. With uniform shading the facets are extremely emphasised. With Gouraud shading the facets are still over empathised while with Phong shading the facets have become smoothed over. It is possible that our shading algorithms do not give the same perceptual benefit that would normally accrue from shading (for further explanation see Ware 1999). This is something that should be considered carefully when designing three-dimensional interfaces.

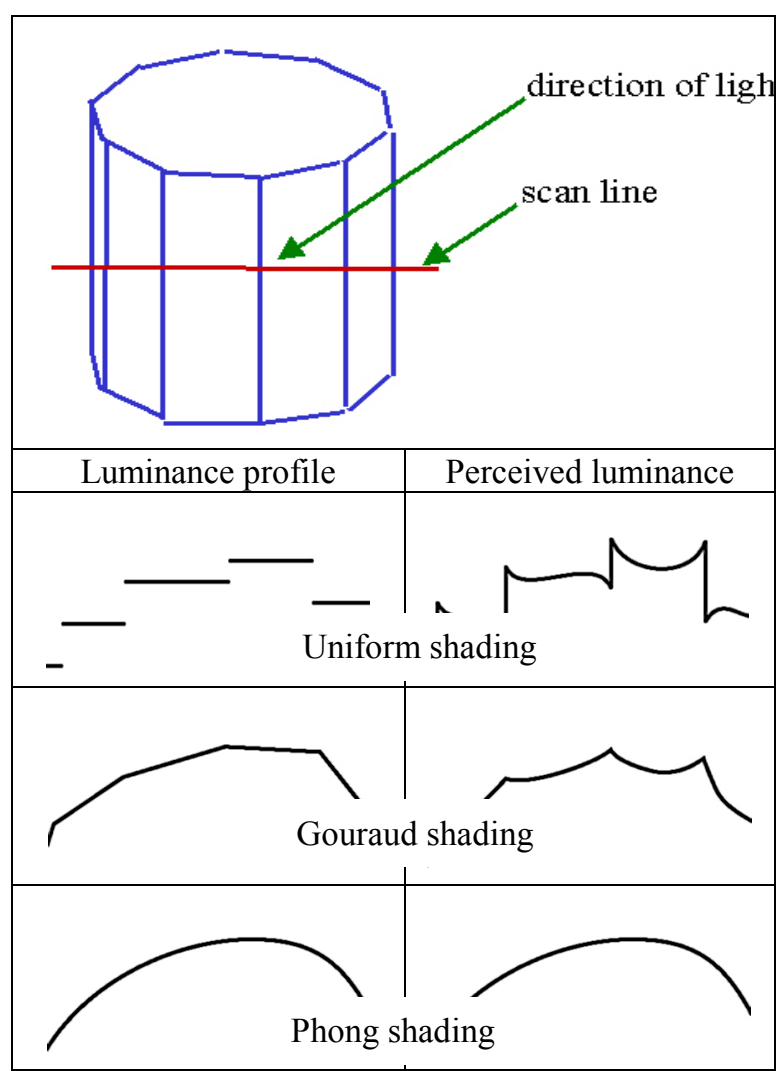

Figure 11: Difference in luminance and perceived luminance from three shading algorithms: uniform, Gouraud and Phong (adapted from Ware 2000, page 82)

\section{DISCUSSION}

According to cognitive science literature the ability to read shape from shading is a pre-attentive ability. "The human visual system is capable of quickly and accurately establishing three dimensions from variations in luminance [shading] only" (Ramachandran 1988). It is probable that this ability to recognise shape from shading is one of the most primitive abilities. Ware (1993) points out that distinguishing shape from shading is part of what he terms a sensory language, which bridges cultures and does not have to be learned. One would think that such a low-level visual routine would be perfect for our purposes and provide us with a method to make distortions explicit. In contrast to all this in our study the effects of shading as a visual cue are counter intuitive. For results as surprising as this one needs to consider possible causes. Some of the suspicions we have are:

- Gouraud shading may not be an adequate substitute for actual shading. If people are not seeing the addition as shading it may simply have the effect of darkening the colours.

- Looking at a 3D image on a flat screen is not the same as viewing $3 \mathrm{D}$ objects in the real world. We need to be careful about assuming that all abilities will transfer.

\section{CONCLUSIONS}

All of the visual cues significantly improved people's ability to locate lenses in both accuracy and speed. By far the most useful and the most preferred cue was the grid. While the shading cue did help in locating the lenses, there were some surprising results with the use of shading in that it did not seem to help the participants in the task of ordering the lenses according to relative magnification. Even combining the grid with the shading did not bring the results to quite as good as the grid alone. The grid alone helped people to perform better overall. We suspect that the reason the grid performed so well overall was that the curved grid lines were easy to read and because to the similarity to the more familiar cartographic grid. It was interesting to find out that the shading cue did not actually provide as much useful information for our participants. This study has demonstrated the effectiveness of using visual cues to aid in comprehension of distortions. It has also raised several questions about the robustness of the use of shading on a computer screen.

\section{Acknowledgements}

This research was supported in part by Intel Inc. and National Sciences and Engineering Research Council of Canada (NSERC). We would like to thank S. Greenberg for helpful suggestions throughout the project. We would also like to acknowledge all the participants in the study.

\section{References}

Bartram, L., \& Ho A., \& Dill, J., \& Henigman F. The continuous zoom: A constrained fisheye 
technique for viewing and navigating large information spaces. In UIST'95: Proceedings of the ACM Symposium on User Interface Software and Technology, pp. 207-215, 1995.

Carpendale, M. S. T., Cowperthwaite, D. J. \& Fracchia, F. D. Three-Dimensional Pliable Surfaces: For effective presentation of visual information. In UIST'95: Proceedings of the ACM Symposium on User Interface Software and Technology, pp. 217-226, 1995.

Carpendale, M. S. T., Cowperthwaite, D. J. \& Fracchia, F. D. Making Distortions Comprehensible, In IEEE Symposium on Visual Languages, pp. 36-45, 1997.

Foley, J. D., van Dam, A., Feiner, S. K., Hughes J. F. Computer Graphics: Principles and Practice. Addison-Wesley Publishing Company. 1996.

Furnas, G. W. Generalized fisheye views. In Proceedings of the ACM Conference on Human Factors in Computer Systems: CHI'86, pp. 16-23, 1986.

Hamel, J. \&, Michel, R., \& Strothotte, T., Visibility through inaccuracy: Geometric distortions to cluttering in route maps. In Proceedings of the Central European Conference on Computer Graphics, pp. 123-132, 1996.

Hollands, J. C. \& Carey, T. T., \& McCann, C. A. Presenting a graphical network: A comparison of performance using fisheye and scrolling views. In Designing and Using Human-Computer Interfaces and Knowledge Based Systems, pp. 313-320, 1989.

Kuederle, O., Inkpen, K. M., Atkins, S., \& Carpendale, M.S.T. Interacting with Image Sequences: Detail-in-Context and Thumbnails. In Proceedings of Graphics Interface, 2001.

Leung, Y. K., \& Apperley, M. D., A review and taxonomy of distortion-oriented presentation techniques. ACM Transactions on ComputerHuman Interaction, vol. 1(2) pp.126-160, 1994.

Leung, Y.K., Spence, R., \& Apperley, M.D. Applying bifocal displays to topological maps. International Journal of Human-Computer Interaction, 7(1), pp. 79-98, 1995.

Meister, D., Lost in computer space. International Journal of Human-Computer Interaction, vol.1(1) pp. 5-21, 1989.

Misue, K., \& Eades, P., \& Lai, W. \& Sugiyama, K. Layout adjustment and the mental map. Journal of Visual Languages and Computing, vol. 6(2) pp.183-210, 1995.
Noik, E. G., Space of Presentation Emphasis Techniques for Visualising Graphs. In the Proceedings of Graphics Interface'94. pp. 225233, 1994.

Plaisant, C., Carr, D., and Shneiderman, B., Imagebrowser Taxonomy and Guidelines for Developers. IEEE Software, 12(2), pp. 21-32, March 1995.

Ramachandran, V. S. Perception of shape from shading. Nature, vol. 331(14), pp.163-166, 1988.

Sarkar, M. \& Brown, M. H. Graphical fisheye views. Communications of the ACM, vol. 37(12), pp. 7384, 1994.

Sarkar, M. \& Snibbe, S., \& Tversky, O. J., \& Reiss, S. P. Stretching the rubber sheet: A metaphor for viewing large layouts on small screens. In Proceedings of the ACM Symposium on User Interface Software and Technology: UIST'93, pp. 81-91, 1993.

Schaffer, D., \& Zuo, Z., \& Bartram, L., \& Dill, J., \& Dubs, S., \& Greenberg, S. \& Roseman, M. Comparing fisheye and full-zoom techniques for navigation of hierarchically clustered networks. In Proceedings of Graphics Interface, pp. 87-96, 1993.

Spence, R., \& Apperly, M. Database navigation: an office environment for the professional. Behaviour and Information Technology, vol. 1(1), pp. 43-54, 1982.

Storey, M.-A. D., \& Müller, H. A. Graph layout adjustment strategies. In the Proceedings of the Third Symposium on Graph Drawing, pp. 487499, 1995

Storey, M.-A. D., Wong, K., Müller, H. A., How do program understanding tools affect how programmers understand programs? In Proceedings of the $4^{\text {th }}$ Working Conference on Reverse Engineering (WCRE97), pp. 12-21, 1997.

van der Heyden, J., K. Inkpen, K., Atkins, S., \& Carpendale, M.S.T.. A User Centred Task Analysis of Interface Requirements for MRI Viewing. In the Proceeding of Graphics Interface, pp. 18-26, 1999.

Ware, C., The foundations of experimental semiotics: a theory of sensory and conventional representation. Journal of Visual Languages and Computing, vol. 4, pp. 91-100, 1993.

Ware, C., Information Visualization: Perception for design. Morgan Kaufmann, 2000. 